\title{
The Infrared Thermal Image and Citokine Il-6 in the Affective Diagnosis of Patients with Non-Communicable Chronic Diseases
}

\author{
David A Rodríguez Medina*1, Benjamín Domínguez Trejo², Patricia Cortés Esteban², Irving Cruz \\ Albarrán ${ }^{3}$, Luis A Morales Hernández ${ }^{3}$, Gerardo Leija Alva ${ }^{4}$, Sandra Vergara Aguirre ${ }^{5}, 0 m a r$ Chavarría \\ Santiago ${ }^{1}$, Carolina Carreño Morales $^{1}$, Sara K Pluma Verde ${ }^{1}$ and Li Erandi Tepepa ${ }^{1}$ \\ ${ }^{1}$ Division of Research and Postgraduate Studies, Universidad National Autónoma de México
}

${ }^{2}$ National Medical Center 20 de Noviembre, Institute de Seguridad y Servicios Sociales de los Trabajadores del Estado, Mexico

${ }^{3}$ Postgraduate in Mechatronics, Universidad Autónoma de Querétaro, Mexico

${ }^{4}$ Interdisciplinary Center of Health Sciences, Institute Politécnico National, Mexico

${ }^{5}$ School of Higher Studies Zaragoza, Universidad National Autónoma de México

Received: May 18, 2018; Published: May 23, 2018

*Corresponding author: David A Rodríguez Medina, Division of Research and Postgraduate Studies, Faculty of Psychology, Universidad National Autónoma de México, Mexico

\begin{abstract}
Peripheral temperature is a useful measure for the evaluation of sympathetic/parasympathetic activity. A non-invasive tool that allows evaluating the peripheral temperature of a patient without contact with the skin is the infrared thermal image (iTF). Various negative affective states (pain, anxiety, depression, anger, alexithymia, social isolation) increase sympathetic activity (decreasing peripheral temperature) and thereby increase pro-inflammatory cytokines, such as interleukin 6 (IL-6) which has been linked to affective alterations; while relaxation promotes parasympathetic activity (increasing peripheral temperature). We present the bio psychosocial evaluation of six patients with different concentrations of IL-6 with diagnoses of chronic non communicable diseases, their iTF pre-post psycho physiological intervention in relaxation and their psychological profile for affective diagnosis. Patients with a higher level of peripheral temperature (hands, nose) or ability to increase it, showed a psychosocial profile without emotional disturbances. In contrast, the patients with the highest level of IL-6 concentration showed the lowest peripheral temperature in the hands and did not manage to increase it after the relaxation strategy. The iTF allows assessing the autonomic activity associated with various affective states. If the patient increases his peripheral temperature after relaxation, he has a better clinical prognosis than the patient who does not.
\end{abstract}

Keywords: Thermography; Interleukin 6; Relaxation; Non-Communicable Chronic Diseases

\section{Introduction}

Non communicable chronic diseases (NCCD) alter the bio psychosocial functioning of people. The physiological alterations of each disease generate autonomic dysfunctions and negative affective states, which increase the pro-inflammatory activity. The increase of pro-inflammatory interleukins influences the central and autonomic nervous system. For its non-invasive evaluation, it is possible to consider the level of concentration of interleukin 6 (IL-6) salivary, and autonomic measurements such as the infrared thermal image (iTF), which allows to evaluate the temperature of a patient without contact with their skin. Several studies have evaluated the effect of the affective state on the peripheral tem perature: the negative affective states (stress, depression, anxiety, fatigue, pain, loneliness) increase the sympathetic tone of peripheral vasoconstriction by lowering the facial temperature and hands [1] and increase inflammatory activity such as IL-6 [2]. Even exposure to negative affective stimuli decreases the nasal temperature [3-5] and increases (tension) in the frontal muscles and chin [6]. On the other hand, to reduce psycho physiological stress, exercise, transdermal nor epinephrine neuro modulation has been shown to reduce stress by increasing peripheral temperature (facial and hands) [7] and relaxation strategies [8]. In this way, autonomic regulation is useful for the affective diagnosis in patients with NCCD, 
in whom, due to the nature of their pathology; autonomic activity and inflammatory response are increased (cancer, arthritis, chronic pain, among others).

\section{Materials and Methods}

We used validated psychometric measures in the target population, as well as the collection of a salivary sample of IL- 6 and its processing according to the guidelines of Salimetrics ${ }^{\circledR}$. Each patient was fasting. We also use the FLIR A310 infrared thermal camera placed one meter away from each patient.

\section{Case Presentation}

We present the thermal psycho physiological evaluation and its level of IL- 6 of 4 patients with non communicable chronic diseases (Multiple myeloma, hyperthyroidism and breast cancer) with dif- ferent emotional functioning: patient 1 with pain and mild anxiety and high level of alexithymia; patient 2 with high level of pain, level of anxiety, alexithymia and mild loneliness; patient 3 without psychological distress; and patient 4 with a high level of pain, control of anger and mild alexithymia. All participants gave their informed consent based on the Helsinki declaration. Based on [8], we replicated the psycho physiological evaluation according to the level of IL-6 concentration in patients with chronic, non-communicable diseases. Figure 1 shows the psycho physiological evaluation and intervention protocol and its effect on peripheral temperature (pre-post relaxation strategies by diaphragmatic breathing). The Figure 2 shows the facial temperature of a 35-year-old woman and a 69-year-old man clinically healthy, with Doctorate education, but different concentration of salivary IL-6.

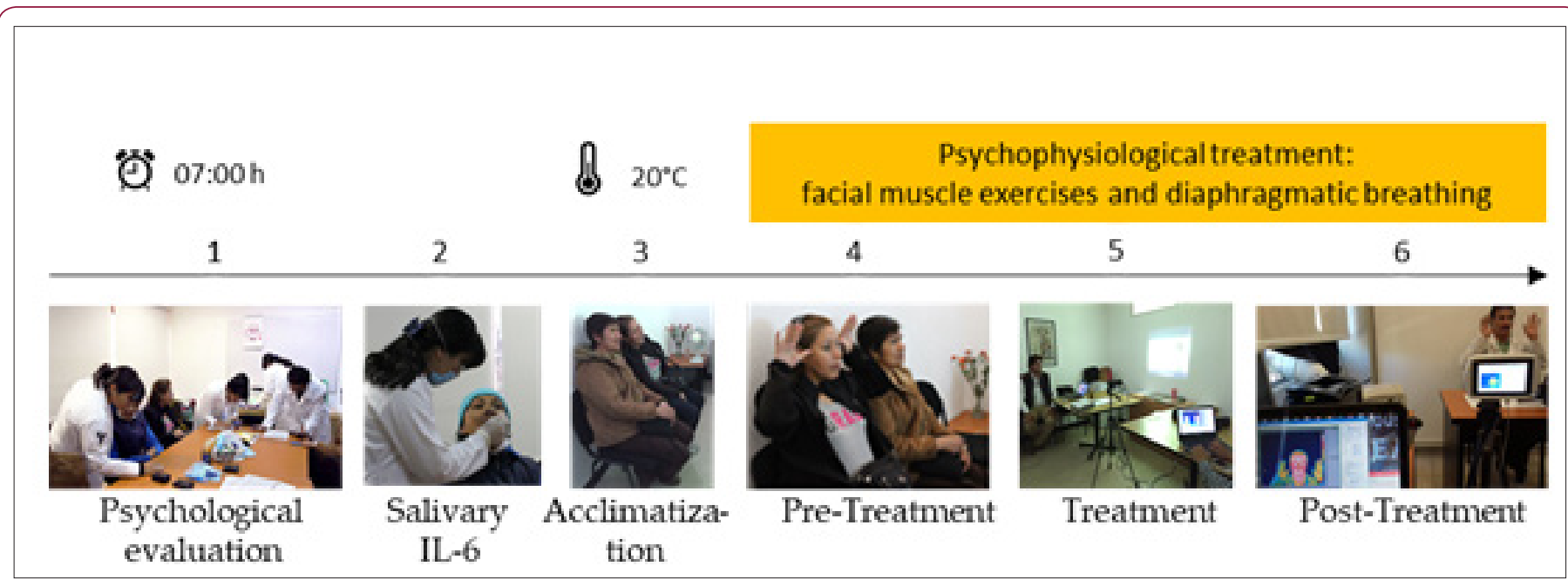

Figure 1: Evaluation and intervention protocol. 1) Psychological evaluation; 2) Collection of salivary IL-6; and 3) Evaluation and psychophysiological intervention monitored with infrared thermal image.

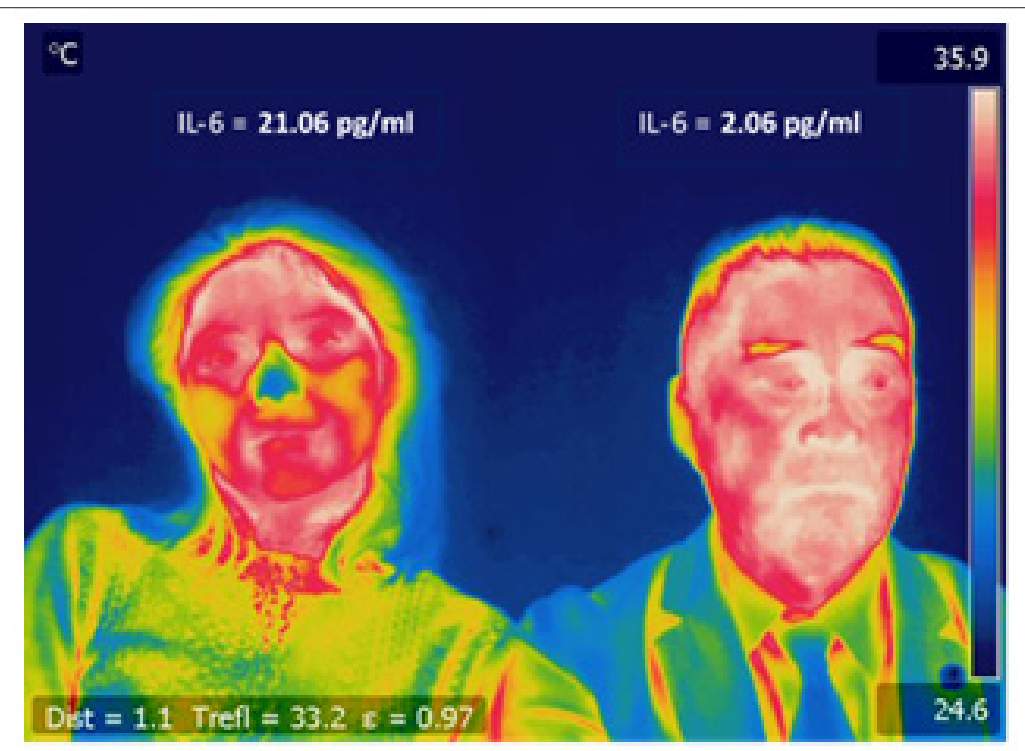

Figure 2: Infrared thermal facial image and concentration level of salivary IL-6 in clinically healthy volunteers. The participant whose nasal temperature is low and the concentration level of IL-6 is high presented a level of sub-clinical anxiety. 


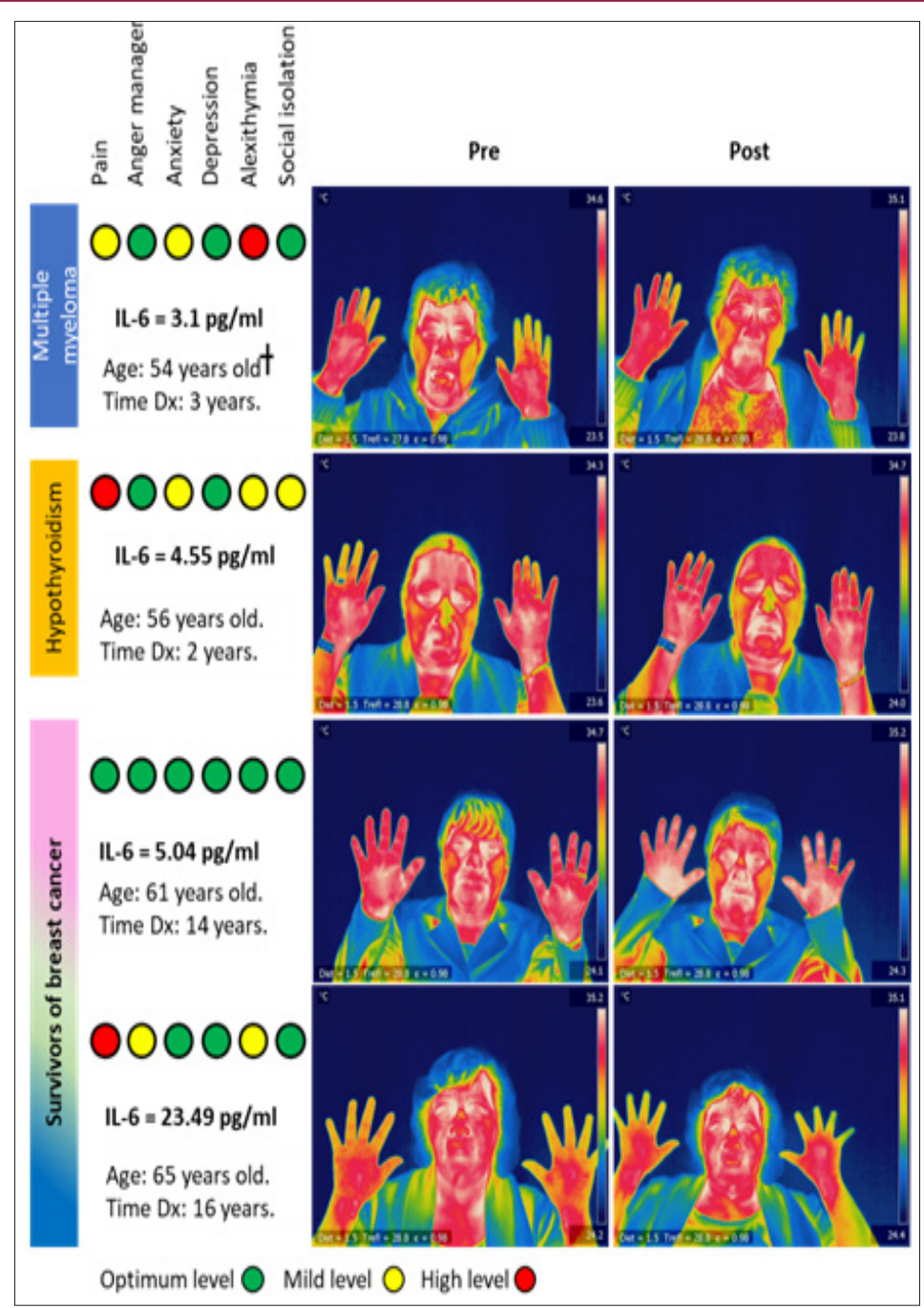

Figure 3: Biopsychosocial evaluation in patients with NCTS pre-post psychological intervention (10 minutes). The colored circles indicate the level of each psychosocial scale: a high level is marked in red, a low level in yellow, while the green color marks a level without clinical attention problems.

\section{Results}

Figure 3 shows the iTF pre-post diaphragmatic breathing, its concentration of IL-6, and its level of psychological distress. The results allow visualizing increases of peripheral temperature in the hands and decreases of temperature in the forehead of patients 1,2 and 3 whose level of IL- 6 is considered low [8]. While patient 4 with a high IL-6 level did not manage to modify its temperature.

\section{Discussion}

The level of concentration of IL-6 influences the ability to relax (increase in temperature in hands and nose) $[8,9]$. The clinical cases presented correspond to patients with non communicable chronic diseases with the purpose of incorporating biomarkers to the psychological evaluation whose purpose is the study of bio psychosocial mechanisms that influence the development and clinical management of the disease. Sympathetic activity increases during negative affective states, such as those explored in the bio psychosocial profile of patients with NCCD. This activity can be monitored during the psychological session: if the patient manages to modify his peripheral temperature, he/she has greater psychological resources to face his/her NCCD. If they cannot increase their peripheral temperature, it is necessary to explore the level of inflammation to channel it with the attending physician.

\section{Acknowledgment}

This research had resources from PAPIIT: IN304515, UNAM. We also thank Gabriela Pérez García, Beatríz Gómez González and Gabriela Hurtado Alvarado.

\section{References}

1. Ioannou S, Gallese V, Merla A (2014) Thermal infrared imaging in psychophysiology: Potentialities and limits. Psychophysiology 51(10): 951-963.

2. Louat K, Berenbaum F (2015) Fatigue in chronic inflammation - a link to pain pathways. Arthritis Research \& Therapy 17(1): 254.

3. Kano F, Hirata S, Deschner T, Behringer V, Call J (2016) Nasal temperature drop in response to a playback of conspecific fights in chimpanzees: A thermo-imaging study. Physiology \& Behavior 155: 83-94. 
4. Salazar López E, Domínguez E, Juárez V, De la Fuente J, Meins A, et al. (2015) The mental and subjective skin: Emotion, empathy, feelings and thermography. The mental and subjective skin: Emotion, empathy, feelings and thermography. Consciousness and Cognition 34: 149-62.

5. Kosonogov V, De Zorzi L, Honoré J, Martínez Velázquez E, Nandrino J, et al. (2017) Facial thermal variations: A new marker of emotional arousal. Plos One 12: 1-15.

6. Rodríguez Medina D, Cruz I, Domínguez B, Morales Hernández L, Leija G (2017) Psycho physiological facial thermal assessment of the relaxation in a patient with osteoarthritis. Pan American J of Med Thermo logy 3(1): 33-36.

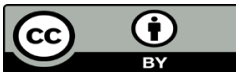

This work is licensed under Creative Commons Attribution 4.0 License

Submission Link: https://biomedres.us/submit-manuscript.php
7. Tyler W, Boasso A, Mortimore H, Silva R, Charlesworth J, et al. (2015) Transdermal neuromodulation of noradrenergic activity suppresses psychophysiological and biochemical stress responses in humans. Sci Rep 5(5): 1-17.

8. Rodríguez Medina DA, Domínguez Trejo B, Cortés Esteban P, Cruz Albarrán IA, Morales Hernández LA, et al. (2018) Bio psychosocial Assessment of Pain with Thermal Imaging of Emotional Facial Expression in Breast Cancer Survivors. Medicines 5(2): 1-16.

9. Lasselin J, Kemani M, Kanstrup M, Olsson G, Axelsson J, et al. (2016) Lowgrade inflammation may moderate the effect of behavioral treatment for chronic pain in adults. J Behav Med 39(5): 916-924.

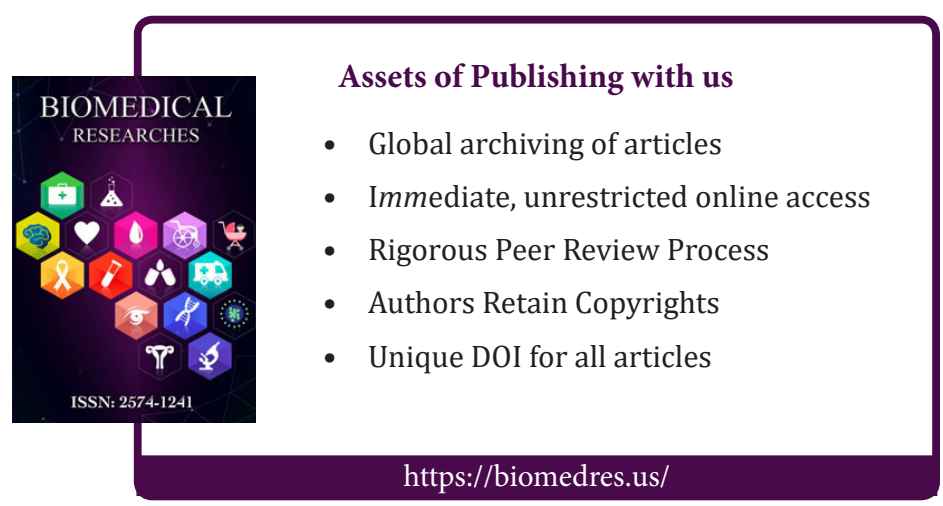

